\title{
Sensitivity and specificity of median nerve ultrasonography in diagnosis of carpal tunnel syndrome
}

This article was published in the following Dove Press journal:

International Journal of General Medicine

27 January 2012

Number of times this article has been viewed

\author{
Mohammad Yazdchi' \\ Mohammad Kazem \\ Tarzemani ${ }^{2}$ \\ Haleh Mikaeili ${ }^{3}$ \\ Hormoz Ayromlu' \\ Hooman Ebadi' \\ 'Neuroscience Research Center, \\ ${ }^{2}$ Department of Radiology, \\ ${ }^{3}$ Tuberculosis and Lung Disease \\ Research Center, Tabriz University \\ of Medical Sciences, Tabriz, Iran
}

Correspondence: Haleh Mikaeili

Tuberculosis and Lung Disease

Research Center, Tabriz University

of Medical Sciences, Tabriz, Iran

Tel +98 4I I 3378093

Fax +98 4I 13378093

Email dr.h.mikaeili@gmail.com
Background: Although controversial, recent studies have demonstrated advantages of sonographic techniques in the diagnosis of carpal tunnel syndrome (CTS). The purpose of this study was to assess the utility of median nerve ultrasonography in the diagnosis of CTS in Iranian patients.

Methods: Ninety patents with clinically suspected CTS were studied. Based on gold standard electromyography/nerve conduction velocity studies, wrists with CTS were divided into three groups on the basis of severity of CTS, ie, mild, moderate, and severe. In addition, both sides of the wrist were examined using sonography. Transverse images of the median nerve were obtained and median nerve cross-section areas were measured at three levels, ie, immediately proximal to the carpal tunnel inlet, at the carpal tunnel inlet, and at the carpal tunnel outlet. Furthermore, flexor retinaculum thickness was evaluated.

Results: The mean age of the studied patients was $48.52 \pm 12.17$ years. Median values of the median nerve cross-section at the carpal tunnel inlet, carpal tunnel outlet, and proximal carpal tunnel significantly differed between the wrists with and without CTS $(P<0.05)$. Comparisons between the CTS groups (mild, moderate, and severe) and non-CTS wrists demonstrated that the median cross-sections of median nerve at the carpal tunnel inlet, carpal tunnel outlet, and inlet proximal carpal tunnel were significantly greater in the severe CTS group than in the other three groups $(P<0.05)$. The results showed that the median nerve cross-section at the three levels of carpal tunnel could only fairly differentiate severe CTS from other cases.

Conclusion: The present study demonstrated that median nerve ultrasonography cannot replace the gold standard test (nerve conduction velocity) for the diagnosis of CTS because of low overall sensitivity and specificity, although it might provide useful information in some patients.

Keywords: median nerve, ultrasonography, carpal tunnel syndrome, diagnosis

\section{Introduction}

Carpal tunnel syndrome (CTS) affects approximately $8 \%$ of the population worldwide and results in median nerve compression in the wrist. CTS is diagnosed on the basis of clinical signs and symptoms and is confirmed by an electrodiagnostic study, which is the most reliable method currently available. ${ }^{1}$ However, sonography could be an alternative method for the diagnosis of CTS, but the precise diagnostic value of this method is controversial. ${ }^{2}$ Polykandriotis et al reviewed 30 patients using ultrasound as the method for CTS diagnosis. ${ }^{3}$ Kwon et al used sonography to demonstrate that the cross-section of the median nerve in the carpal tunnel inlet is significantly greater in CTS patients than in control groups. The optimal cutoffs were a median nerve crosssectional area of $10.7 \mathrm{~mm}^{2}$ at the tunnel inlet, and this resulted in $63 \%$ sensitivity 
and $66 \%$ specificity. $^{4}$ These numbers indicate a relatively high rate of false negative and false positive results with ultrasonography. Furthermore, Wong et al studied 120 suspected CTS patients, and demonstrated average median nerve cross-sections in the carpal tunnel inlet and outlet of $9 \mathrm{~mm}^{2}$ and $12 \mathrm{~mm}^{2}$, respectively. The sensitivity and specificity rates were $94 \%$ and $65 \%$, respectively. ${ }^{5}$ Thus, the latter study revealed at least good sensitivity of median nerve sonography in the diagnosis of CTS. Although controversial, several recent studies have demonstrated advantages of sonography techniques for the diagnosis of CTS. The aim of the present study was to assess the utility of median nerve sonography in the diagnosis of CTS patients in Iran.

\section{Methods}

In a cross-sectional study, 90 patents with clinically suspected CTS aged 15-65 years who had symptoms such as paresthesia and painful hands, particularly at night, were studied from July 2008 to December 2009. On admission, patients underwent clinical examination, hand muscle atrophy was assessed, and Tinel's and Phalen's tests were performed. Patients with traumatic fractures and wrist injuries resulting in anatomical changes that would disrupt ultrasonography were excluded. Demographic information including gender, age, occupation, previous history of disease, pregnancy in female patients, medication history, laboratory test results, results of electromyography/nerve conduction velocity (EMG/NCV) studies, and severity of involvement were recorded. In addition, imaging findings were recorded to evaluate the sensitivity and specificity of sonography. The study protocol was approved by the institutional ethics committee. Written informed consent was obtained from all patients prior to the study.

The EMG/NCV standard median nerve apparatus TOENINNIES NeuroScreen Plus was utilized and EMG/ NCV studies were performed. Wrists with and without CTS were distinguished, and involved wrists were divided into three groups on the basis of the severity of CTS, ie, mild, moderate, and severe. Patients included in the mild group showed median distal sensory latency of greater than 3.5 milliseconds, or the sensory latency difference of $>0.5$ milliseconds between the median and ulnar nerves. The moderate group had median nerve sensory nerve action potential amplitude reduction (less than 50\% compared with the normal side or less than $25 \mathrm{mV}$ ) or median nerve distal motor latency prolonged by more than 4.5 milliseconds. The severe group had severely reduced compound muscle action potential amplitude of the median nerve (less than 50\% compared with the normal side or below $4 \mathrm{mV}$ ) or demonstrated denervation of muscles upon needle examination.

Both sides (right and left wrists) were examined by a blinded radiologist using sonography (Aloka 3500 Pro Probe Linear 7.5-10 mHz). Transverse images of the median nerve were obtained, and median nerve cross-section areas were measured at three levels, ie, immediately proximal to the carpal tunnel inlet, at the carpal tunnel inlet, and at the carpal tunnel outlet. Furthermore, flexor retinaculum thickness was evaluated.

Data were presented as the mean \pm standard deviation, and median and interquartile range or percentage, whenever appropriate. Statistical analysis was performed using SPSS for Windows (v 12.0; SPSS Inc, Chicago, IL). KruskalWallis test and post hoc Mann-Whitney U test were used for analysis. A receiver-operator characteristic curve analysis was performed to obtain critical values allowing the utility of ultrasound examination in prediction of severe CTS. A $P$ value less than 0.05 was considered to indicate statistical significance.

\section{Results}

The mean age of the studied patients was $48.52 \pm 12.17$ (range 23-65) years; 68 were female and 22 were male. Ninety patients (180 wrists) were evaluated. Sixty-five patients had bilateral involvement, 19 involved the right hand, and six involved the left hand. The average duration of involvement was $24.4 \pm 39.5$ months (15 days to 20 years). The most common complaints were numbness and paresthesia ( $\mathrm{n}=86,95.6 \%)$, hand pain $(\mathrm{n}=85,94.4 \%)$, pain during the night ( $\mathrm{n}=80,88.9 \%)$, and hand atrophy $(\mathrm{n}=16,17.8 \%)$.

In 25 wrists $(13.9 \%)$, no evidence of median nerve involvement was found. The EMG/NCV results indicated that 34 (18.9\%), 99 (55\%), and 22 (12.2\%) wrists had mild, moderate, or severe involvement, respectively. KruskalWallis testing revealed statistically significant differences in median values of the median nerve cross-section at the carpal tunnel inlet, carpal tunnel outlet, proximal carpal tunnel between the wrists with median nerve entrapment, and wrists without nerve involvement $(P=0.006,0.001$, and 0.002 , respectively). There was no statistically significant difference between the two groups (wrists with and without CTS) with respect to flexor retinaculum thickness $(P=0.420$, Table 1).

Post hoc comparisons between the CTS groups (mild, moderate, and severe) and non-CTS wrists demonstrated that the median cross-sections of median nerve at the carpal tunnel inlet, carpal tunnel outlet, and inlet proximal carpal tunnel 
Table I Median nerve cross-section areas at different levels and flexor retinaculum thickness across groups

\begin{tabular}{|c|c|c|c|c|c|}
\hline & No CTS & Mild CTS & Moderate CTS & Severe CTS & CTS (total) \\
\hline Carpal tunnel inlet (cross-section, mm²) & $11.10 \pm 3.11$ & $|I .4| \pm 2.56$ & $12.40 \pm 4.01$ & $15.10 \pm 4.74$ & $12.56 \pm 3.99$ \\
\hline Carpal tunnel outlet (cross-section, $\mathrm{mm}^{2}$ ) & $10.15 \pm 2.87$ & $10.47 \pm 2.20$ & $11.25 \pm 3.29$ & $13.86 \pm 4.04$ & $11.44 \pm 3.35$ \\
\hline Proximal carpal tunnel (cross-section, $\mathrm{mm}^{2}$ ) & $10.24 \pm 2.60$ & $10.65 \pm 2.41$ & $11.12 \pm 3.17$ & $13.93 \pm 3.73$ & $11.42 \pm 3.26$ \\
\hline Flexor retinaculum thickness $(\mathrm{mm})$ & $1.30 \pm 1.92$ & $1.39 \pm 1.72$ & $1.71 \pm 2.38$ & $1.86 \pm 2.65$ & $1.66 \pm 2.28$ \\
\hline
\end{tabular}

Abbreviation: CTS, carpal tunnel syndrome.

were significantly greater in the severe CTS group than the other three groups $(P<0.05)$. There were no differences in this regard between the control wrists and wrists with mild CTS $(P>0.1)$. Although wrists with moderate CTS tended to have higher median cross-sections of median nerve at the carpal tunnel inlet and outlet compared with control and mild CTS wrists, the differences did not reach statistical significance $(0.05<P<0.1)$. There was no statistically significant difference between the groups with respect to flexor retinaculum thickness $(P>0.1)$.

In receiver-operator characteristic curve analysis, the area under the curve was insignificant for mild-to-moderate CTS $(P>0.05)$. Receiver-operator characteristic curve analysis was also performed to assess the utility of ultrasound examination in prediction of severe CTS. The results showed that the median nerve cross-section at the three levels of the carpal tunnel could fairly predict severe CTS from other cases (Figure 1). With a cutoff point of $\geq 12.5 \mathrm{~mm}^{2}$ for the median nerve cross-section at the carpal tunnel inlet, the sensitivity and specificity of ultrasonography was $71.4 \%$ and $59.1 \%$, respectively (area under the curve $=0.678$, $P=0.008)$. With a cutoff point of $\geq 11.5 \mathrm{~mm}^{2}$ for the median nerve cross-section at the carpal tunnel outlet, the sensitivity and specificity of ultrasound examination was $76.2 \%$ and $57.1 \%$, respectively (area under the curve $=0.724$, $P=0.001$ ). Finally, with a cutoff point of $\geq 11.5 \mathrm{~mm}^{2}$ in the proximal carpal tunnel inlet, the sensitivity and specificity of ultrasonography was $76.2 \%$ and $56.5 \%$, respectively (area under the curve $=0.713, P=0.002$ ).

\section{Discussion}

In the present study, the efficacy of ultrasound for the diagnosis of CTS was evaluated. Electrodiagnostic studies (EMG/ $\mathrm{NCV}$ ) were used as gold standard diagnostic procedures. ${ }^{6,7}$

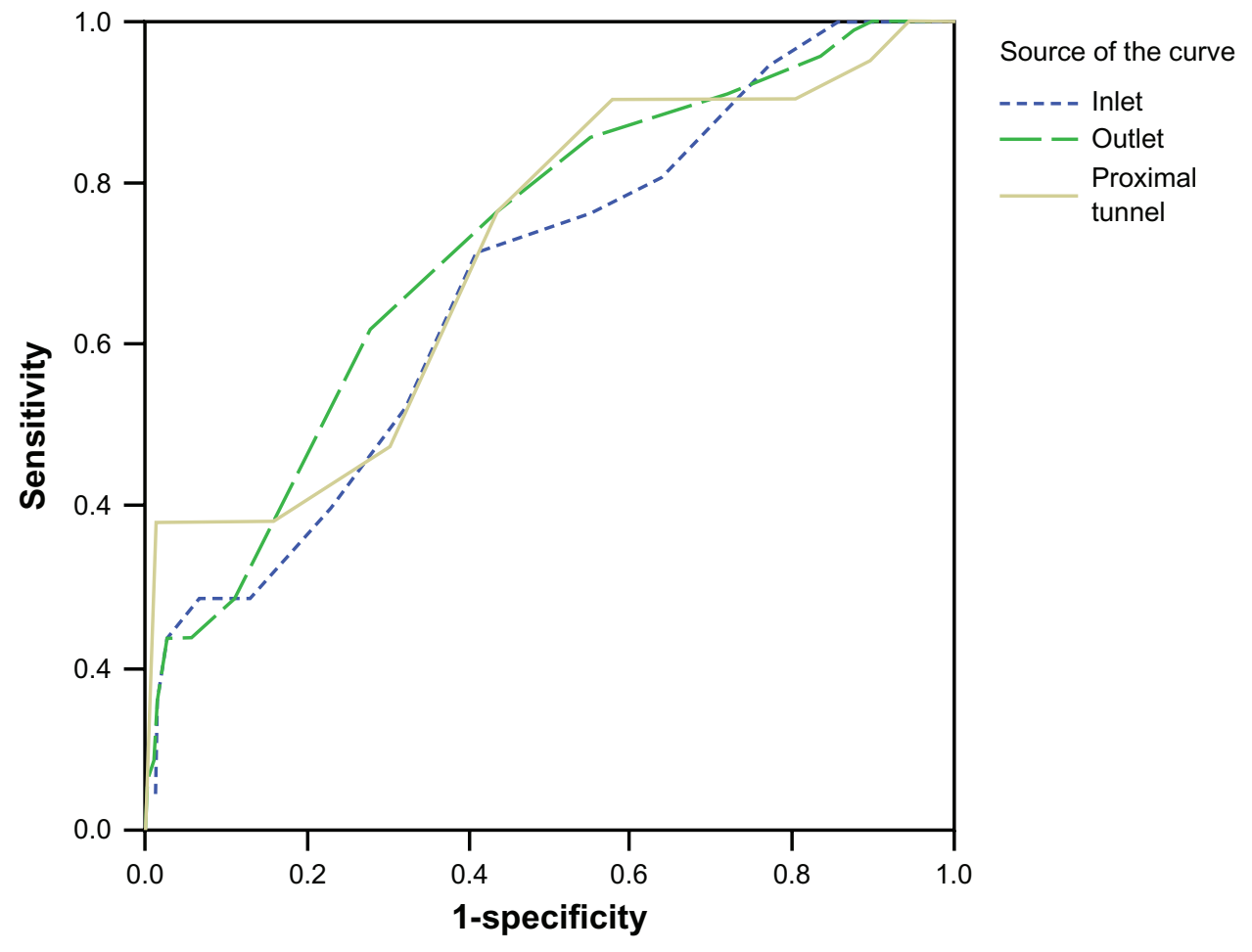

Figure I Receiver operating characteristic curve for median nerve cross-section at the three levels of carpal tunnel to predict the case of severe carpal tunnel syndrome from other cases. 
Although no statistically significant difference was found between CTS and control wrists with respect to median nerve cross-section areas at different carpal levels, the median values for wrists with severe CTS were significantly higher than for other groups. However, receiver-operator characteristic curve analysis showed that although median nerve ultrasonography could fairly predict severe CTS (area under the curve, $0.50-0.75$ ), the overall sensitivity and specificity of ultrasonography was low.

Recent studies have demonstrated that the average crosssection of the median nerve in the outlet and inlet of the carpal tunnel was significantly greater in CTS patients than in control groups. Furthermore, these studies demonstrated that the best cutoff point for the median nerve cross-section was $9-15 \mathrm{~mm}^{2}$, and that sensitivity and specificity varied between $70 \%$ and $89 \%$ and between $57 \%$ and $97 \%$, respectively. ${ }^{5-12}$

Ashraf et al studied 70 CTS cases and 80 individuals without CTS (controls) in Iran. The average diameter of the median nerve in the experimental group was significantly higher than in the control group. It has been reported that the optimal cutoff point in this field is more than $9.3 \mathrm{~mm}^{2}$ (sensitivity $80 \%$ and specificity $77 \%$ ). ${ }^{10}$ Our findings are in agreement with the results of previous studies. The average of these parameters was higher in the patients than in the control group, but these differences were not statistically significant. In addition, low sensitivity and specificity was evident using these cutoff points. Unlike the results of previous studies, we demonstrated nonsignificant differences between the two groups for the mean of median nerve cross-sections in different areas. This could be due to the low number of samples included in this study and the fact that 25 cases were included in the control group; some studies included only control subjects without complaint or history of CTS. In this study, the control group was selected from CTS patients suffering from CTS only on one side. Therefore, the normal side of the inflamed median nerve could be asymptomatic and undetected using electrodiagnostic criteria. This could result in the absence of significant differences despite apparent differences between the two groups.

To the best of our knowledge, the role and influence of CTS severity in patients has not been studied. In the current study, the average thickness of the median nerve measured at three different points was significantly greater in the severe CTS group (criteria EMG/NCV) than in the other CTS groups and hands without CTS; differences between mild and moderate CTS cases and the control group were not significant. Higher sensitivity and specificity $(76.19 \%$ and $72.08 \%$, respectively) were detected in the proximal tunnel inlet and outlet (cutoff points $11.5 \mathrm{~mm}^{2}$ and $12.5 \mathrm{~mm}^{2}$, respectively). Therefore, the use of ultrasound in such patients should be limited to distinguishing severe CTS from milder forms. However, further investigations are required in view of the small sample size used in the present study. In addition, due to the nature of CTS, median nerve swelling levels are located inside and outside the carpal tunnel. Therefore, determining a definitive cutoff point is impracticable. ${ }^{5}$

In the present study, the thickness of the flexor retinaculum was evaluated, and despite the aforementioned limitations of the study, these findings were comparable with those reported by Wong et al. ${ }^{8}$ Keleş et al demonstrated that thickness of the flexor retinaculum can be used in the diagnosis of CTS. In this study, flexor retinaculum thickness $>3.7 \mathrm{~mm}$ (sensitivity $71.4 \%$ and specificity $55 \%$ ) was considered significant in the evaluation of CTS patients. ${ }^{6}$

The results presented herein demonstrate that ultrasonography could not replace the gold standard test (NCV) for diagnosis of CTS (especially mild and moderate CTS, that accounts for most cases) because of low overall sensitivity and specificity CTS. However, further studies with a larger sample size, selection of normal individuals as the control group, and following up patients with false positive results are required to confirm the usefulness of sonography for the diagnosis of CTS. Ultimately, although median nerve ultrasonography findings at wrist may differ, at least in wrists with severe nerve entrapment, from controls, our results did not show that ultrasonography is of sufficiently good sensitivity and specificity in diagnosis of CTS.

\section{Disclosure}

The authors report no conflicts of interest in this work.

\section{References}

1. Visser LH, Smidt MH, Lee ML. High-resolution sonography versus EMG in the diagnosis of carpal tunnel syndrome. J Neurol Neurosurg Psychiatry. 2008;79:63-67.

2. Mondelli M, Filippou G, Gallo A, Frediani B. Diagnostic utility of ultrasonography versus nerve conduction studies in mild carpal tunnel syndrome. Arthritis Rheum. 2008;59:357-366.

3. Polykandriotis E, Premm W, Horch RE. Carpal tunnel syndrome in young adults - an ultrasonographic and neurophysiological study. Minim Invasive Neurosurg. 2007;50:328-334.

4. Kwon BC, Jung KI, Baek GH. Comparison of sonography and electrodiagnostic testing in the diagnosis of carpal tunnel syndrome. J Hand Surg Am. 2008;33:65-71.

5. Wong SM, Griffith JF, Hui AC, Lo SK, Fu M, Wong KS. Carpal tunnel syndrome: diagnostic usefulness of sonography. Radiology. 2004;232: 93-99.

6. Keleş I, Karagülle Kendi AT, Aydin G, Zöğ SG, Orkun S. Diagnostic precision of ultrasonography in patients with carpal tunnel syndrome. Am J Phys Med Rehabil. 2005;84:443-450. 
7. Swen WA, Jacobs JW, Bussemaker FE, de Ward JW, Bijlsma JW. Carpal tunnel sonography by the rheumatologist versus nerve conduction study by the neurologist. $J$ Rheumatol. 2001;28:62-69.

8. Wong SM, Griffith JF, Hui AC, Tang A, Wong KS. Discriminatory sonographic criteria for the diagnosis of carpal tunnel syndrome. Arthritis Rheum. 2002;46:1914-1921.

9. Yesildag A, Kutluhan S, Sengul N, et al. The role of ultrasonographic measurements of the median nerve in the diagnosis of carpal tunnel syndrome. Clin Radiol. 2004;59:910-915.
10. AshrafAR, Jali R, Moghtaderi AR, Yazdani AH. The diagnostic value of ultrasonography in patients with electrophysiologically confirmed carpal tunnel syndrome. Electromyogr Clin Neurophysiol. 2009;49:3-8.

11. Sarría L, Cabada T, Cozcolluela R, Martínez-Berganza T, García S. Carpal tunnel syndrome: usefulness of sonography. Eur Radiol. 2000; 10:1920-1925.

12. Lee D, van Holsbeeck M, Janevski PK, Ganos DL, Ditmars DM, Darian VB. Diagnosis of carpal tunnel syndrome: ultrasound versus electromyopathy. Radiol Clin North Am. 1999;37:859-872.

International Journal of General Medicine

\section{Publish your work in this journal}

The International Journal of General Medicine is an international, peer-reviewed open-access journal that focuses on general and internal medicine, pathogenesis, epidemiology, diagnosis, monitoring and treatment protocols. The journal is characterized by the rapid reporting of reviews, original research and clinical studies across all disease areas.

\section{Dovepress}

A key focus is the elucidation of disease processes and management protocols resulting in improved outcomes for the patient.The manuscript management system is completely online and includes a very quick and fair peer-review system. Visit http://www.dovepress.com/ testimonials.php to read real quotes from published authors.

Submit your manuscript here: http://www.dovepress.com/international-journal-of-general-medicine-journal 